\section{Tratamiento ortopédico funcional de los maxilares y ortodóntico en paciente con síndrome del cromosoma X- frágil. Reporte de caso}

\author{
Functional orthopedic jaw and orthodontic \\ treatment in a patient with fragile- $X$ \\ syndrome. A case report
}

\begin{abstract}
Resumen
El síndrome X frágil (SXF) es un trastorno ligado al cromosoma X, en el brazo largo $\mathrm{Xq} 27.3$, que provoca diversas alteraciones como problemas de conducta, deficiencia intelectual, macroorquidia, pabellones auditivos grandes y prominentes, paladar profundo y ojival, prognatismo mandibular, maloclusión y anomalías dentarias. El objetivo de este informe fue presentar el caso clínico de una paciente de 16 años con SXF, leucodermia, que se sometió a un tratamiento ortopédico funcional de los maxilares para la corrección del apińamiento dentario. En el examen clínico se observaron timidez, ansiedad, inestabilidad emocional, trastornos conductuales esporádicos asociados a discapacidad intelectual leve, alteraciones craneofaciales y oclusales. Luego del estudio, evaluación radiográfica panorámica y trazados cefalométricos, se decidió instalar un dispositivo ortopédico funcional de maxilar, tipo Pistas Planas Indirectas, para posterior tratamiento ortodóncico correctivo. Bien al inicio del tratamiento se observó dificultad de comprensión y colaboración por parte de la paciente y su responsable (madre) y, luego de 5 meses, aún con mejoras en las funciones estomatognáticas, se inició el tratamiento ortodóntico con dispositivo fijo, el que fue concluido luego de dos ańos. El éxito del tratamiento de ortopedia funcional de los maxilares y/u ortodóntico, principalmente en el SXF, se basa en el abordaje comportamental y motivación en todas las etapas del tratamiento por el profesional, así como en un ambiente familiar colaborativo.
\end{abstract}

Palabras clave: Síndrome del cromosoma X frágil; Odontología; Deficiencia intelectual; Ortodoncia correctiva; Atención dental para personas con discapacidades (fuente: DeCS BIREME).

\begin{abstract}
The Fragile-X Syndrome (FXS) is a disorder linked to X chromosome, on the long arm Xq27.3, causing several changes such as behavioral problems, intellectual disability, macroorchidism, large and prominent auricles, deep and ogival palate, mandibular prognathism, increased mandibular angle, malocclusion, and dental anomalies. The objective was to present a case of a 16-year-old patient with FXS, leukoderma, submitted to orthopedic functional maxillary treatment to correct dental crowding. In general, clinical examination, behavioral changes such as shyness, anxiety, emotional lability, sporadic
\end{abstract}

\section{Caso Clínico}

\author{
Aida Sabbagh-Haddad 1,a, Patrícia Valéria Cunha \\ Georgevich ${ }^{1, b}$, Monica Salfatis ${ }^{1, c}$, Denise Sabbagh \\ Haddad ${ }^{2, \mathrm{~d}}$ \\ ${ }^{1}$ Práctica privada \\ 2 Universidade de São Paulo (USP), Faculdade de Odon- \\ tologia (FO), São Paulo, Brasil. \\ a Doctora en Diagnóstico Bucal. \\ ${ }^{\mathrm{b}}$ Magister en Ortodoncia y Ortopedia Funcional de los \\ Maxilares. \\ ${ }^{c}$ Magister en Ortodoncia y Ortopedia Facial. \\ d Investigador Posdoctoral.
}

\section{Correspondencia:}

Aida Sabbagh-Haddad : aidasabbagh@hotmail.com Al. dos Maracatins, 426 - cj. 912 - CEP: 04089-000; São Paulo-SP; Brasil

ORCID: 0000-0001-6769-8480

\section{Coautores:}

Patrícia Valéria Cunha Georgevich: odontovaleria@uol. com.br

ORCID: 0000-0002-6495-655X

Monica Salfatis: salfatismonica@gmail.com ORCID: 0000-0002-9487-2648

Denise Sabbagh Haddad: deniseshaddad@hotmail.com ORCID: 0000-0001-7053-0881

Editor:

Donald Ramos Perfecto

Universidad Nacional Mayor de San Marcos, Perú.

Conflicto de intereses: los autores declaran no tener conflictos de interés.

\section{Fuente de financiamiento: autofinanciado.}

\section{Recibido: 24/11/20}

Aceptado: 03/02/21

Publicado: 01/07//21 
disturbances of behavior associated with mild mental disabilities were remarkable. After panoramic radiographic evaluation and cephalometric tracings, it was decided to install the functional orthopedic appliance of the jaws, Indirect Flat Planes type, for later corrective orthodontic treatment. At beginning of treatment, there was a difficulty in understanding and collaborating, not only from the patient's side but also from the mother's. After five months, even with the improvement in stomatognathic functions, orthodontic treatment with a fixed appliance was started, which was concluded after two years. Success of functional and / or orthodontic jaw orthopedics treatment, especially in FXS, is based on behavioral approach and motivation in all stages, by the professional as well as a collaborative family environment.

Keywords: Fragile X syndrome; Dentistry; Intellectual disability; Corrective orthodontics; Dental care for disabled (source: MeSH NLM).

\section{Introducción}

El síndrome de X frágil (SXF), también conocido como síndrome de Martin-Bell, es una condición de causa genética, por lo tanto, de carácter hereditario dominante, que se produce debido a alteraciones en el gen FMR1 (Retraso Mental Frágil tipo -1) ubicado en el cromosoma X.

La denominación de SXF se relaciona a una región frágil, localizada en la porción distal del brazo largo del cromosoma X, específicamente en Xq27.3 $3^{1,2,3}$. Esta característica se puede verificar mediante el examen del cariotipo, siempre que se utilice la técnica específica para buscar sitios frágiles ${ }^{4}$. Sin embargo, a partir de los años 90, el diagnóstico se puede confirmar mediante el examen molecular, que en los hombres se realiza de manera indirecta a través del método de PCR, mientras que, en las mujeres, es necesario utilizar los métodos de Southern Blot e Hibridación para el diagnóstico final del síndrome ${ }^{5}$.

La alteración que afecta al gen $F M R 1$ es una expansión de tres nucleótidos CGG (citosina-guanina-guanina) y dependiendo del mayor o menor número de nucleótidos CGG, se pueden apreciar los efectos variables sobre la constitución fenotípica del paciente con el síndrome ${ }^{6,78}$.

Es de gran importancia obtener el diagnóstico correcto con la realización del examen molecular (ADN), con el fin de iniciar un plan de estimulación precoz y tratamiento de los síntomas, así como para realizar el asesoramiento genético.

SFX es considerada la segunda patología más frecuente asociada a la discapacidad intelectual, de etiología genética, siendo superada apenas por el Síndrome de Down ${ }^{1,2}$. Por ello, se desconoce su incidencia real en la población, pero respecto a su prevalencia existen diferencias poblacionales, pudiendo ser muy alta, oscilando entre $1 / 4,000$ hombres y $1 / 8,000$ mujeres afectadas por la mutación completa; en cambio, para los portadores de la premutación, las cifras son mayores, con 1/259 mujeres y $1 / 379$ hombres ${ }^{9}$.

Así, la manifestación clínica más importante es la discapacidad intelectual provocada por la ausencia de la proteína FMRP en el cerebro de estos individuos ${ }^{10,11}$. En los hombres suele ser grave (Coeficiente intelectual (CI) entre 20 y 35) o predominantemente moderado (CI entre 35 y 50) y en aproximadamente un tercio de las mujeres, discapacidad intelectual leve o limítrofe. Existe un descenso en los resultados de las pruebas de evolución del CI, siendo el período más llamativo el inicio de la pubertad (11 a 15 ańos), lo que sugiere la existencia de un proceso degenerativo continuo ${ }^{1,4}$.

Asimismo, en el cuadro clínico del SXF, además de la discapacidad intelectual, se observa que existe una tríada clásica, formada por macroorquidismo, pabellones auriculares grandes y prominentes y un rostro alargado y estrecho. La longitud del rostro es mayor debido a la rotación de la mandíbula hacia abajo ${ }^{12}$ y un aumento en el ángulo de la mandíbula en un promedio de 8 grados ${ }^{13}$. Los cambios fenotípicos son más evidentes en la pubertad, sirviendo de advertencia a los cirujanos dentistas, por tener un aspecto facial dismórfico, pues, además de los ya mencionados, presentan el tercio medio de la cara hipoplásica $(89 \%)$, frente alta y prominente y rebordes supraorbitarios prominentes ${ }^{1,2}$.

Otras características son la hiperextensión de las articulaciones de los dedos de las manos, pie plano, alteraciones del tercer metacarpiano de la falange, displasia del tejido conectivo y piel elástica; también se han informado problemas oculares como estrabismo, atrofia óptica y miopía ${ }^{1,2}$; ocasionalmente puede haber otitis media recurrente, aumento de peso y perímetro cefálico al nacer ${ }^{1,3}$, nariz larga, escaso vello corporal, piel fina y aterciopelada con estrías, tortícolis, cifoescoliosis ${ }^{3} \mathrm{y}$ prolapso de la válvula mitral en el $8 \%{ }^{1}$.

Las anomalías neurológicas más comunes encontradas en el SXF son atrofia de la región posterior del vermis cerebeloso y agrandamiento del ventrículo IV ${ }^{3,14}$. El electroencefalograma muestra cambios en aproximadamente el $60 \%$ de los casos, ocurriendo principalmente en pacientes que sufren de convulsiones ${ }^{3}$.

La epilepsia puede ocurrir en el 10 al 20\% de los pacientes con SXF, siendo el tipo rolándico el más frecuentemente observado en la infancia, debido a una deficiencia de la proteína FMRP ${ }^{15,16}$.

Las características conductuales presentes en el SXF incluyen poco o escaso contacto visual, agitar la mano, contacto físico defensivo e impulsividad, además de hiperactividad, agresividad, ansiedad y automutilación ${ }^{1,2,17,18}$. 
Estos pacientes pueden tener un perfil conductual, en algunos aspectos, similar al de los individuos con autismo, sin embargo, la mayoría no cumple con todos los criterios para el diagnóstico de autismo, por lo que existe controversia en la literatura entre la asociación de SXF y autismo ${ }^{1,2,16}$.

Para la realización del tratamiento odontológico, el abordaje de los pacientes con SXF se vuelve muy complejo y difícil debido a la presencia de discapacidad intelectual, discapacidad o falta de comunicación (lenguaje) y también por cambios de comportamiento ${ }^{19,20}$; en este último se pueden observar algunas manifestaciones clínicas similares a las observadas en individuos con Trastorno del Espectro Autista (TEA), donde en la mayoría de los casos el tratamiento odontológico solo se realizará previa sedación o anestesia general. Sin embargo, las personas con TEA pueden beneficiarse del uso de métodos de comunicación alternativos conocidos como el Treatment and Education of Autistic and related Communication-handicapped children (Tratamiento y educación de niños autistas y niños con problemas de comunicación relacionados) (TEACCH®), Picture Exchange Communication System (Sistema de comunicación de intercambio de imágenes) (PECS®) y del Programa Son-Rise $®$ para realización del tratamiento dental.
El presente caso clínico tiene como objetivo describir las características físicas y bucales, así como los aspectos conductuales de una paciente con SXF, analizar y alertar a los odontólogos sobre las posibilidades del tratamiento ortopédico funcional instituido y ortodóncico de los maxilares, mostrando y discutiendo los diferentes niveles de comunicación y colaboración durante el tratamiento.

\section{Reporte del caso}

Paciente con síndrome del X Frágil, 16 años, sexo femenino, leucodermia, de estatura alta (Figura 1), con un hermano mayor también con SXF, que acudió a la clínica con la queja principal de "dientes torcidos y que por tal motivo, quisiera usar aparatos ortopédicos".

En el examen clínico general de la paciente se observaron cambios craneofaciales típicos: rostro alargado y estrecho, frente prominente, nariz larga, perfil convexo, mandíbula retraída, pabellones auriculares grandes, órbitas poco profundas, esclerótica azulada, estrabismo leve y miopía (Figura 2). Además, se presentan marcados cambios de comportamiento como timidez, cierta inseguridad, ansiedad, inquietud, labilidad emocional, trastornos esporádicos del comportamiento (terquedad), escaso contacto visual, déficit de atención

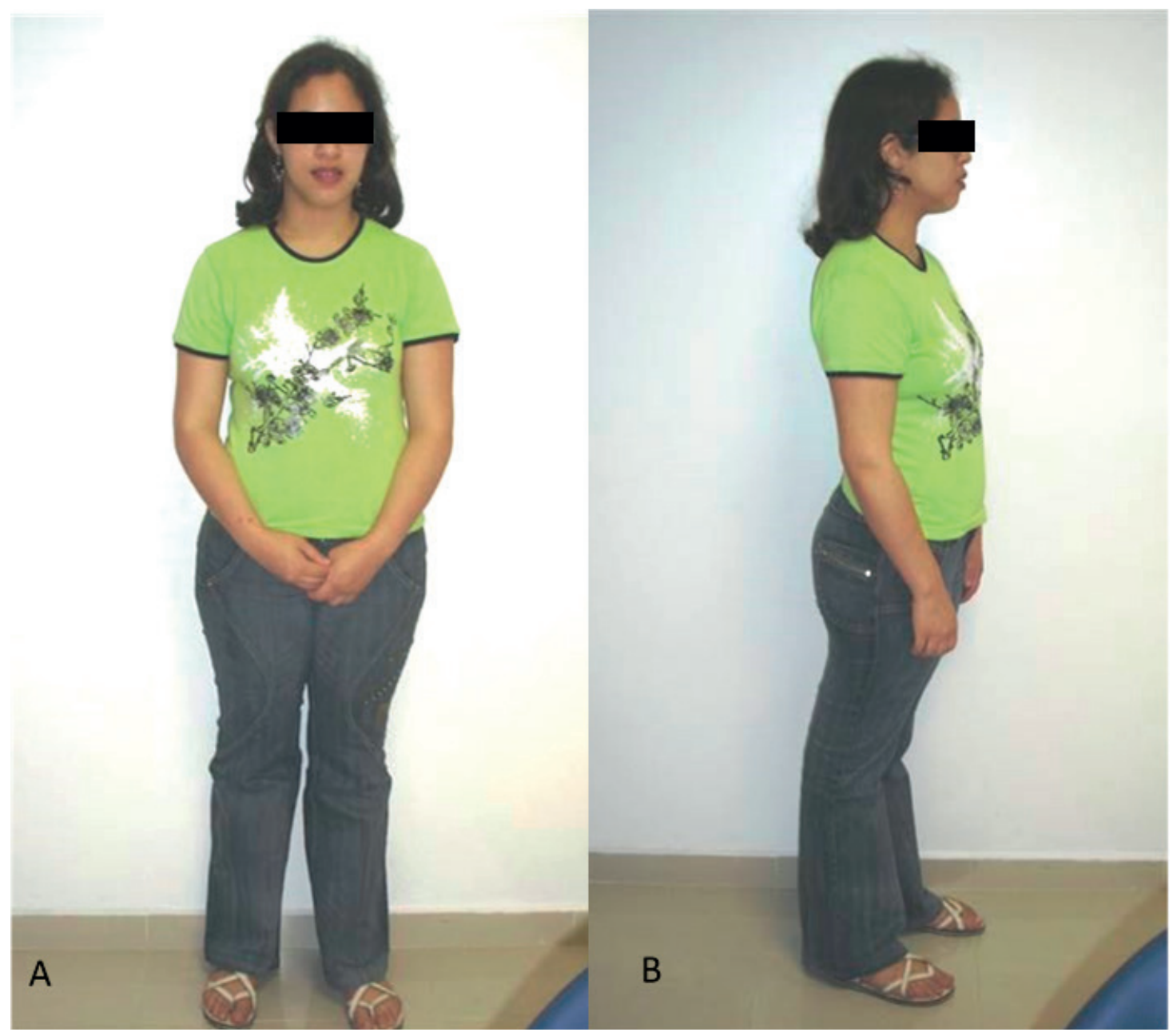

Figura 1. Vista frontal (A) y lateral (B) de la paciente portadora del SXF 
asociado a discapacidad intelectual moderada, determinando dificultades de aprendizaje. Su relación familiar fue descrita como satisfactoria e inestable y el entorno en el que vive carece de la estimulación adecuada que difiere de los estándares educativos. Adquisición del lenguaje y el habla con ligero retraso. Habla infantilizada y regresión de hábitos.

El examen intraoral reveló paladar profundo y ojival, mordida cruzada anterior localizada en el incisivo lateral superior izquierdo, mordida cruzada posterior izquierda, desviación de la línea media y primer molar permanente en normoclusión, caracterizando la clase I de Angle. No se observaron cambios dentales estructurales, de tamaño o numéricos. La paciente mostró una buena higiene bucal, sin problemas gingivales o periodontales, así como dientes afectados por caries (Figuras 3 y 4 ).

El examen radiográfico panorámico reveló que todos los dientes permanentes están erupcionados, a excepción de los terceros molares superiores e inferiores (Figura 4) y, según el estadio de mineralización dentaria de Nolla, estos están avanzados para la edad de la paciente.

El dispositivo de elección para este estudio fue el aparato ortopédico funcional de los maxilares de Pistas Planas Indirectas (Figura 5), indicado según los análisis facial y cefalométrico, cuyos ángulos S-N.A, S-N.B y A-N.B son respectivamente $82,7^{\circ}, 77,8^{\circ}$ y $4,9^{\circ}$.

El mecanismo de acción del aparato funciona cuando las placas se colocan en contacto con los arcos, utilizando también superficies deslizantes que tienen la función de contactar la placa inferior contra la superior, sin causar interferencia dentaria. Además de la función de contacto, las superficies deslizantes también actúan facilitando el movimiento lateral, rehabilitando la ATM, corrigiendo la disoclusión, impidiendo la mesiooclusión y ayudando a superar las oclusiones cruzadas.
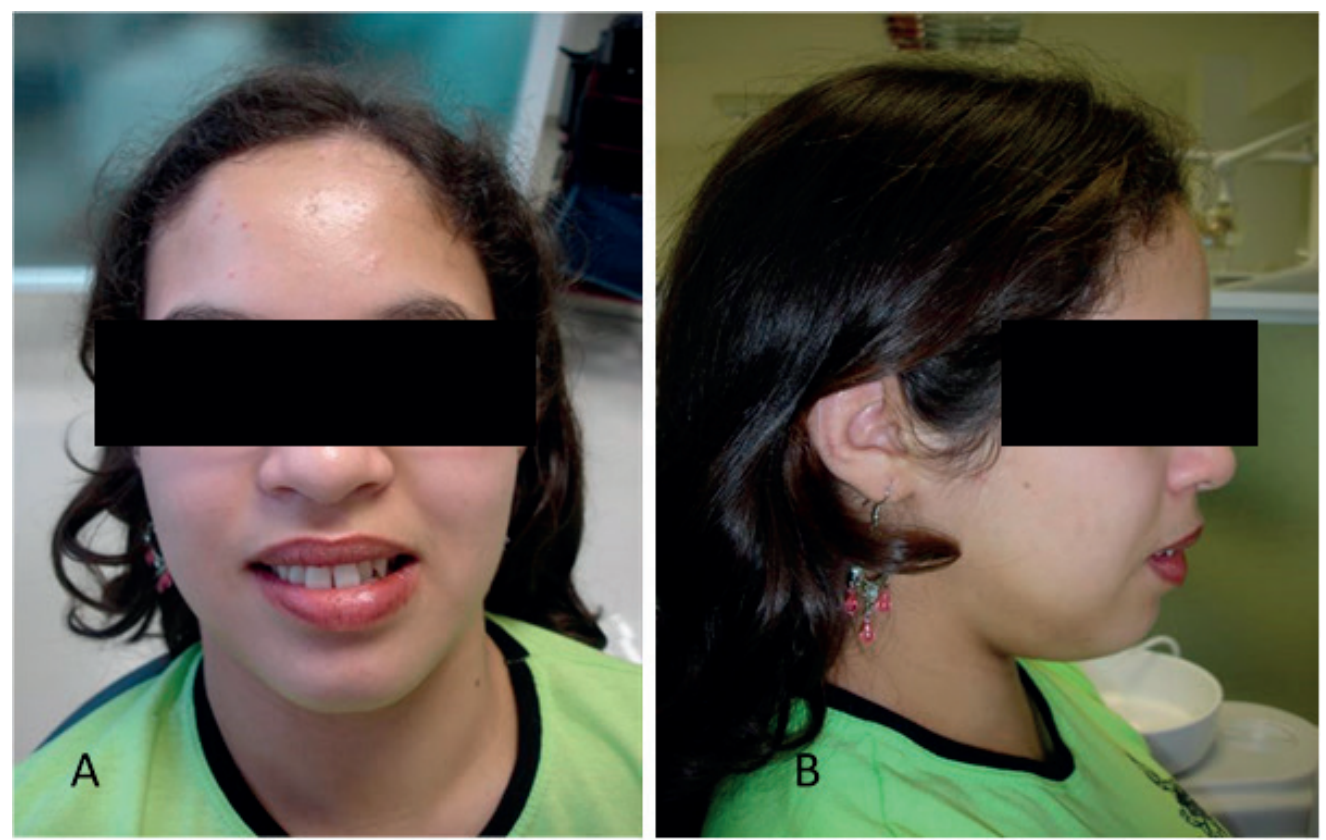

Figura 2 Aspectos faciales de la paciente: (A) rostro largo y estrecho, miopía, leve estrabismo y nariz larga; (B) orejas grandes y prominentes
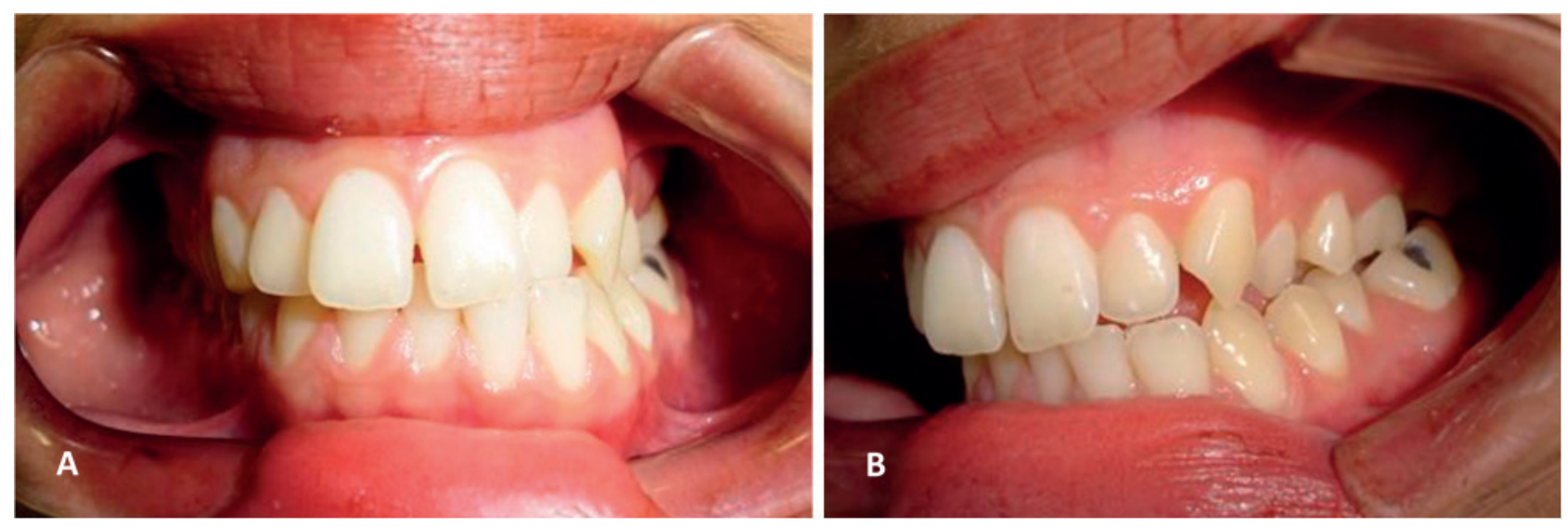

Figura 3. Examen intrabucal: desvío de la línea media (A) y mordida cruzada posterior (B) 


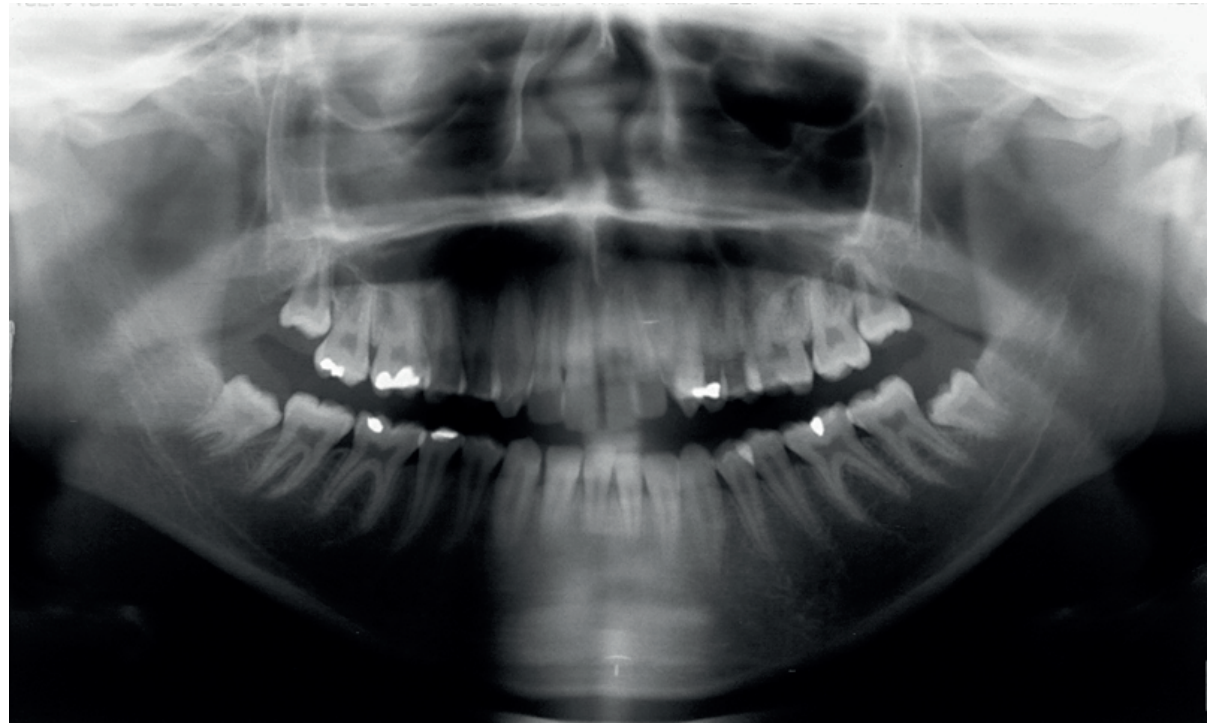

Figura 4. Radiografía panorámica de la paciente.
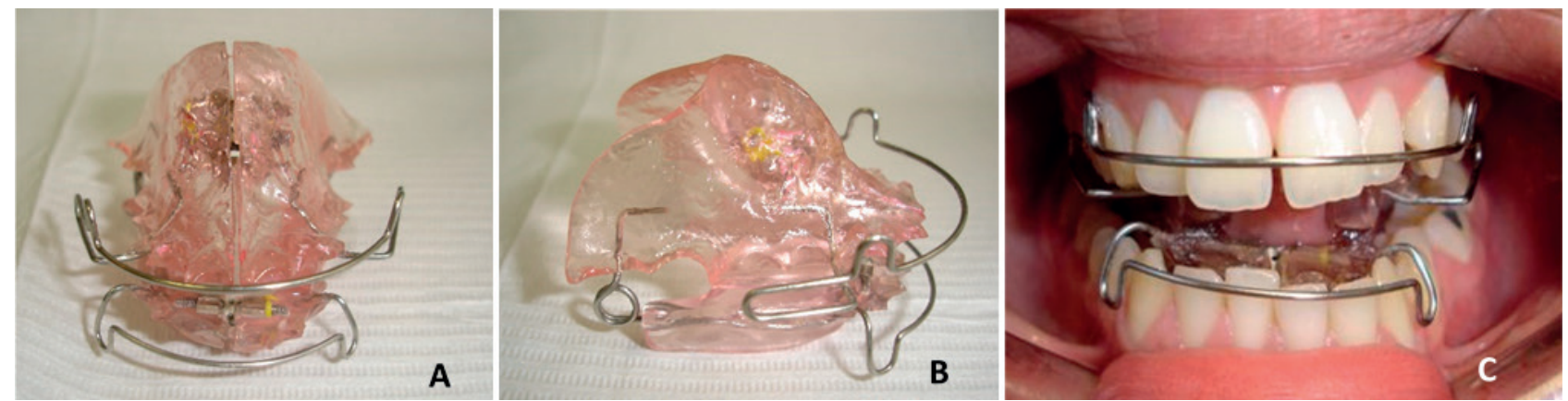

Figura 5. Vista frontal (A) y lateral (B) del dispositivo ortopédico funcional de los maxilares Pistas Planas Indirectas. Dispositivo ortopédico instalado (C).

El dispositivo Pistas Planas descrito en este informe está indicado en casos de normoclusión (Clase I de Angle), apińamiento superior e inferior, mordida cruzada anterior y posterior.

Resultados del tratamiento. La paciente inicialmente se mostró colaboradora en el tratamiento ortopédico funcional de los maxilares, en lo que respecta a la preparación de documentación ortodóncica, radiografías e impresiones para la obtención del modelo de estudio.

Durante la consulta para la instalación del aparato (Figura 5), se transmitieron a la madre las instrucciones de uso y activación del dispositivo (en cada consulta de mantenimiento se activaron los tornillos expansores del dispositivo). Después del primer mes de uso, la paciente estaba un poco triste e irritada con su madre, quien la animó a usar el aparato. A pesar de las quejas de la paciente con respecto al dispositivo, la línea media, que se desvió hacia la izquierda, comenzó a mostrar una leve mejoría.

A los dos meses, la paciente regresó a la clínica muy irritada, con un comportamiento muy agresivo. La madre también informó estar muy descontenta, diciendo que su hija ya no quería usar el dispositivo, debido al dolor y la incomodidad al hablar. Hablamos tanto con la madre como con la paciente sobre las ventajas y desventajas en relación con el uso del dispositivo ortopédico funcional, tratando de persuadir a la paciente. La madre mostró signos de no querer que su hija usara más el dispositivo, siendo muy poco colaboradora y alentadora, indicando que la hija se quejaba mucho de dolor y malestar en cada sesión y que presentaba un comportamiento agresivo como resultado de este hecho. Sin embargo, luego de nuestro abordaje conductual explicando los beneficios del dispositivo al cuarto mes de uso, la paciente acudió a la consulta muy descontenta, quejándose mucho, manifestando que sentía dolor cada vez que se activaba el dispositivo y que este le apretaba los dientes. Se observó que el incisivo lateral superior izquierdo ya estaba en proceso de descruzamiento y la línea media continuaba mostrando cambios positivos. Durante este período, se realizaron cuatro activaciones del tornillo expansor, con una activación cada 30 días.

Después de cinco meses de utilizar el aparato ortopédico funcional para los maxilares de Pistas Planas Indirectas, la paciente regresó a la clínica afirmando que había pasado aproximadamente treinta días sin utilizar el aparato. El examen clínico oral reveló que la evolución obtenida 
por la paciente, en los pocos meses de uso del dispositivo, retrocedió, al presentar nuevamente desviación de la línea media y mordida cruzada del incisivo lateral superior izquierdo.

Ante este hecho, decidimos indicar el tratamiento de ortodoncia mediante aparatología fija (Figura 6). La paciente aceptó mejor este abordaje, aunque en todas las consultas se enfatizó la importancia del aparato fijo, además de motivarla a realizar una adecuada técnica de cepillado. Después de un año y medio se retiró el aparato fijo donde logramos una adecuada oclusión (Figura 7).

\section{Discusión}

El análisis de la literatura científica consultada muestra pocos artículos sobre niñas afectadas por el síndrome del $\mathrm{X}$ frágil. Sin embargo, su riqueza semiológica implica un mejor conocimiento del diagnóstico precoz, que se ve obstaculizado por la variabilidad que presenta $y$, por tanto, en el adecuado abordaje multidisciplinario.

Clínicamente, se encontró que la paciente también presentaba algunas de las alteraciones mencionadas en la literatura, como discapacidad intelectual moderada, orejas grandes ${ }^{1,4,6,16}$, cara alargada y estrecha ${ }^{6,12}$ así como problemas oftalmológicos (miopía y estrabismo leve) y nariz larga ${ }^{3}$. No se observó problemas sistémicos como cardiopatías, manifestaciones epilépticas, alteraciones hematológicas, respiratorias o diabetes mellitus.

En la literatura científica consultada se encontraron pocos estudios que aborden las manifestaciones bucales de este síndrome. Los autores citan el paladar alto y ojival y la mandíbula prominente como las principales características presentes ${ }^{1,17,21,22}$. Sin embargo, la paciente no presentó prognatismo mandibular, lo que es contrario a la literatura. También pueden presentar anodoncia, manchas e hipoplasia del esmalte, anomalías en la forma, macrodoncia y desgaste incisal; también se observaron problemas periodontales, involucrando encía marginal e insertada. En cuanto a la oclusión, predomina la clase I de Angle y la mordida cruzada unilateral y bilateral, cuando se compara con un grupo control de individuos con discapacidad intelectual de causa inespecífica ${ }^{1,2}$. Entre las características bucales predominantes, la paciente también presentaba mordida cruzada unilateral posterior y anterior, con relación molar clase I de Angle ${ }^{1}$. El volumen de la lengua era normal, no caracterizando macroglosia ${ }^{21}$.

Hernandez et al. (2009) caracterizaron el perfil de comportamiento autista de los niños con $\mathrm{X}$ frágil con base en el Manual diagnóstico y estadístico para el trastorno mental, cuarta edición; ellos concluyeron que el trastorno del espectro autista es un subfenotipo diferente en el síndrome de X frágil caracterizado por déficits en la interacción social compleja, con similitudes al trastorno del espectro autista en la población general ${ }^{23}$. Los aspectos conductuales también pueden mitigarse o exacerbarse según los factores ambientales en los que vive el niño, como en su entorno familiar o escolar. Esta investigación puede aportar información importante sobre problemas conductuales de etiología no genética ${ }^{24}$. Los cambios conductuales observados en esta paciente fueron timidez, ansiedad y labilidad

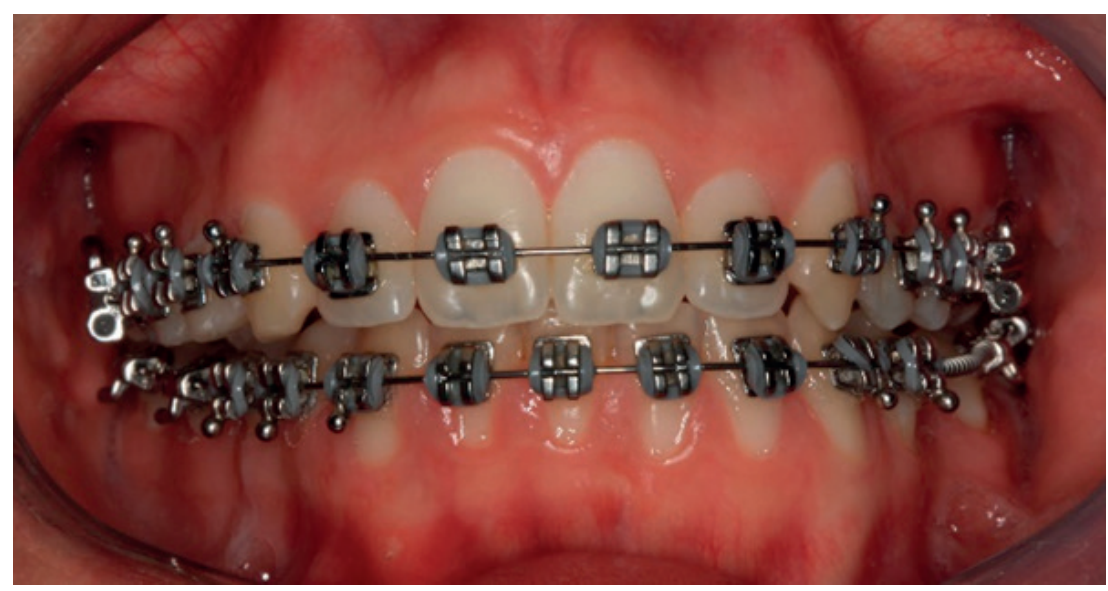

Figura 6. Dispositivo fijo ortodóntico instalado
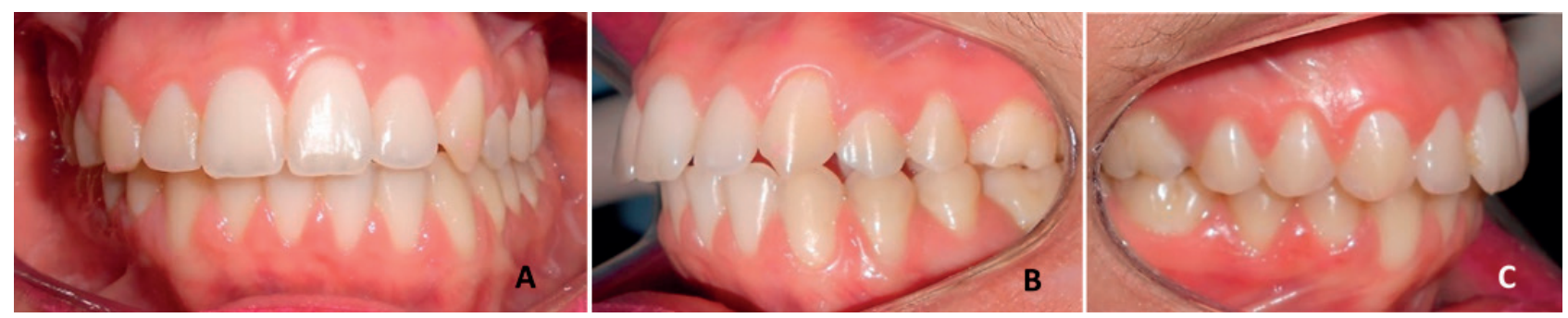

Figura 7. Tratamiento ortodóntico concluido: vista en oclusión frontal (A), lateral izquierda (B) y lateral derecha (C) 
emocional ${ }^{4}$, además de desviar la mirada con desviación de la cabeza cuando es observada ${ }^{1,16,17}$. También mostró cierta apatía emocional e irritabilidad cuando se le contradecía, lo que también es un sello distintivo del síndrome ${ }^{6}$. La paciente era hiperactiva en la clínica y la madre informó que también tenía este comportamiento en el hogar y en la escuela, demostrando ser un importante aspecto conductual del síndrome de $\mathrm{X}$ frágil $^{2,6,22,25}$.

Sabbagh-Haddad (1999, 2007) 1,2 demostró, mediante evaluación radiográfica panorámica, que en el grupo con SXF, al compararlo con el grupo de individuos con discapacidad intelectual de causa inespecífica, existe un avance en la cronología de la erupción dentaria en el Grupo SXF de todos los terceros y segundos molares inferiores; las anomalías dentales más frecuentes fueron los dientes caducos supernumerarios $(2,83 \%)$, dientes permanentes girovertidos $(2,31 \%)$ y la anodoncia $(1,82 \%)$. En otro estudio, en 2016, se comparó un grupo de individuos con SXF con un grupo de individuos normales, pareados por edad (rango de edad de 6 a 17 años), género, incluyendo individuos con dentición decidua, mixta y permanente; el análisis del examen radiográfico panorámico mostró que existe un avance en la cronología de la erupción dentaria, según los criterios de Nolla (1960) de todos los terceros molares y segundos molares inferiores en individuos con SXF; en cuanto a las anomalías dentarias más frecuentes encontradas en la dentición temporal en el SXF fueron las supernumerarias $(2,83 \%)$, en la dentición permanente los dientes girovertidos $(2,31 \%)$ y la anodoncia parcial $(2,31 \%)$. Los autores destacaron la importancia del conocimiento por parte del odontólogo de las posibles alteraciones radiográficas dentales presentes, ayudando en el plan de tratamiento de los pacientes con SXF ${ }^{13}$. En este caso clínico no se observaron alteraciones dentales estructurales, de tamaño o numéricas.

En SXF se observó maduración dentaria precoz donde las etapas de formación y cronologías de erupción dentaria fueron más avanzadas en comparación con la edad ósea y la altura del paciente ${ }^{26}$. Durante la evaluación de la paciente, nuestros hallazgos coincidieron con la literatura científica.

Aunque existen muchas implicaciones que contraindican el tratamiento ortopédico / ortodóntico en pacientes con discapacidad intelectual ${ }^{27,28}$, debido a que la paciente mostró una buena relación y cooperación en las primeras etapas del tratamiento dental ( 4 meses) y la presencia de un mal posicionamiento dental, fue indicado el aparato ortopédico del maxilar de Pistas Planas Indirectas. Durante el tratamiento, a pesar de hablar e instruir al tutor y la paciente sobre los beneficios que se estaban obteniendo, a los 5 meses la paciente no quiso seguir usando este aparato removible quejándose de dolor e incomodidad. En ese momento decidimos indicar y realizar el tratamiento de ortodoncia con la instalación de aparato fijo convencional, donde los objetivos se lograron satisfactoriamente después de un año y medio de instalación. La finalización del tratamiento de ortodoncia no logró una oclusión perfecta como resultado de la cooperación de la paciente, con un resalte aumentado y una relación canina inadecuada. Esto se debió principalmente a las limitaciones de comportamiento inherentes al SXF (que tenían la madre y el paciente), que incluyen problemas de comunicación y deterioro motor. Así, las instrucciones de higiene y cuidado del dispositivo, con la alimentación y uso de gomas elásticas comprometieron en gran medida el avance del caso. La paciente olvidaba usar los elásticos y a menudo mostraba roturas constantes de los brackets, factores que contribuyen en la evolución de la mecánica. En el caso de pacientes portadores de SXF, debido a que tienen discapacidad intelectual y alteraciones en el comportamiento, todas las instrucciones deben ser lo más claras posibles, además de motivar constantemente al paciente.

El reconocimiento del paciente con SXF es importante, ya que además de los cambios cognitivos, físicos, conductuales y comunicativos, muchas veces se asocian con enfermedades sistémicas, las cuales deben ser evaluadas, ya que pueden influir en el plan de tratamiento odontológico, que definirá su desempeño en nivel ambulatorio. Además, la riqueza de su semiología implica un mejor conocimiento del abordaje multidisciplinario y del diagnóstico precoz, que se ve obstaculizado por la variabilidad de esta.

Con base en los resultados obtenidos, podemos afirmar que es fundamental hablar, instruir y animar a los padres y/o cuidadores de personas con necesidades especiales sobre el uso de aparatos ortopédicos funcionales para los maxilares y los beneficios de este tratamiento. En el caso de los portadores de SXF, por presentar discapacidad intelectual, esta instrucción debe llevarse a cabo con la mayor claridad posible, ya que, en este síndrome, por tener una etiología genética, los padres son portadores de esta discapacidad, habiendo, por lo tanto, mayor dificultad de comprensión y colaboración por parte de ellos.

De lo relatado en el caso clínico se concluye que: la discrepancia en la relación maxilomandibular y consecuentemente problemas de oclusión se observan frecuentemente en SXF, determinando la indicación de tratamiento ortodóntico $\mathrm{y} / \mathrm{u}$ ortopédico funcional de los maxilares. Es fundamental instruir adecuadamente a los padres y/o cuidadores del paciente con Síndrome del $\mathrm{X}$ Frágil sobre el uso continuo del aparato ortopédico funcional de los maxilares, así también, es necesaria una relación satisfactoria entre el paciente y sus padres y/o cuidadores para estimularlo y motivarlo durante todo el período de tratamiento hasta alcanzar la meta. Resaltamos que el ambiente familiar desagradable fue un factor importante que dificultó el tratamiento ortopédico funcional de los maxilares de la paciente con Síndrome del X Frágil, requiriendo el establecimiento de un tratamiento de ortodoncia con brackets fijos convencionales, logrando así la corrección dental deseada.

\section{Referencias bibliográficas}

1. Sabbagh-Haddad, A. Aspectos clínicos e radiográficos panorâmicos das manifestaçôes bucais na Síndrome do 
X-frágil (Síndrome de Martin-Bell) [tesis de doutorado]. [São Paulo]: Faculdade de Odontologia da Universidade de São Paulo; 1999. 112 p.

2. Sabbagh-Haddad A. Síndrome do X-Frágil ou Síndrome de Martin-Bell En: Odontología para Pacientes com Necessidades Especiais. SABBAGH-HADDAD, A (Coordenadora). São Paulo: Livraria Santos Editora, 2007. p. 214-228.

3. Pires CAL, Borghetti VHS, Zaffari A Pacheco A, Moro A. Síndrome do X Frágil. Rev Médica HSVP 1999;11(24):69-73.

4. Yonamine SM, Silva AA. Características da comunicação em indivíduos com a síndrome do X frágil. Arq Neuropsiquiatr. 2002;60(4):981-985.

5. Alliende MAR, Curotto BL, Valiende G, Toro J, Santa Maria L, González MR. Diagnostico citogenético-molecular del Síndrome Xq frágil. Rev Chil Tecnol Méd 2007;27(1):1339-1346.

6. Marcantonio SM, Menezes HS, Magdalena N. Síndrome do X frágil: revisão. Revista AMRIGS. 1998;42(4):226231.

7. Mazzocco MM. Advances in research on the fragile X syndrome. Ment Retard Dev Disabil Res Rev. 2000;6(2):96-106.

8. Murray A, Ennis S, Macswiney F, Webb J, Monton NE. Reproductive and menstrual history of females with fragile X expansions. Eur J Hum Genet. 2000;8(4):247-252.

9. Mazzoco MM. Advances in research on the fragile X syndrome. Ment Retard Dev Disabil Res Rev. 2000;6(2):96109.

10. Willemsen R, Olmer R, Otero YDD, Oostra BA. Twin sisters, monozygotic with the fragile $\mathrm{X}$ mutation, but with a different phenotype. J Med Genet 2000;37(8):603-604.

11. Yim SY, Jeon BH, Yang JA, Kim HJ. Fragile X syndrome in Korea: a case series and a review of the literature. J Korean Med Sci. 2008;23(3):470-76.

12. Kulkarni GV, Levine N. Fragile X (Martin-Bell) syndrome. Spec Care Dentist. 1994;14(1): 21-25.

13. Sabbagh-Haddad A, Sabbagh-Haddad D, Michel-Crosato E, Arita ES. Fragile X syndrome: panoramic radiographic evaluation of dental anomalies, dental mineralization stage, and mandibular angle. J Appl Oral Sci. 2016;24(5):518-23.

14. Guerreiro MM, Camargo EE, Kato M, Marques-De-Faria AP, Ciasca SM, Guerreiro CAM, Menezes Netto JR, Moura-Ribeiro MVL. Fragile X Syndrome: clinical eletroencephalographic and neuroimaging characteristics. Arq Neuropsiquiatr 1998;56(1): 18-23.

15. Berry-Kravis E. Epilepsy in fragile X syndrome. Dev Med Chil Neurol. 2002;44(11):724-728.
16. Veiga NF, Toralles MBP. A expressão neurológica e o diagnóstico genético nas síndromes de Angelman, de Rett e do X-frágil. J Pediatr (Rio J). 2002;78(supl. 1): S55-S62.

17. Lachiewicz AM, Dawson DV, Spiridigliozzi GA. Physical Characteristics of Young Boys With Fragile X Syndrome: Reasons for Difficulties in Making a Diagnosis in Young Males. Am J Med Genet 2000;92:229-236.

18. Scully C. Fragile X (Martin Bell) Syndrome. Dent Update. $2002 ; 29(4): 196-198$.

19. Ferrando MT, Banus P, Lopez G. Cognitive aspects in girls with fragile X syndrome. Rev Neurol. 2004;38 Suppl 1:S53-7.

20. Hatton DD, Wheeler A, Sideres J, Sullivan K, Reichardt A, Roberts J, Clark R, Bailey DB. Developmental trajectories of young girls with fragile X syndrome. Am J Intellect Dev Disabil. 2009;114(3):161-171.

21. Magalhães MHCG, Faria ADA, Bueno DF, Pereira MC. Aspectos odontológicos da síndrome do X frágil - relato de dois casos clínicos. RPG. 1998;5(3):192-196.

22. Pandey UB, Phadke SR, Mittal B. Molecular diagnosis and genetic counseling for the fragile $\mathrm{X}$ mental retardation. Neurol India 2004;52:36-42.

23. Hernandez RN, Feinberg RL, Vaurio R, Passanante NM, Thompson RE, Kaufmann WE. Autism spectrum disorder in fragile $\mathrm{X}$ syndrome: a longitudinal evaluation. Am J Med Genet A. 2009;149A(6):1125-37

24. Hessl D, Dyer-Friedman J, Glaser B, Wisbeck J, Barajas RG, Taylor A, Reiss AL. The influence of environmental and genetic factors on behavior problems and autistic symptoms in boys and girls with fragile $\mathrm{X}$ syndrome. Pediatrics. 2001;108(5):E88. DOI: 10.1542/ peds.108.5.e88.

25. Carvajal FI, Quirós BA, Toral FJ, Orriols JJ, Ramos AMJ, Cantalapiedra SA, Rodrígues MJF, Luances PR. Eficacia de un test clínico como preselección de niños com sospecha de síndrome X frágil. An Esp Pediatr 2001;54(4):326-330.

26. Kotilainen J, Pirinen S. Dental Maturity is Advanced in Fragile X Syndrome. Am J Med Genet 1999;83:298301.

27. Bock JJ, Maurer P, Otto C, Fuhemann RA, Schubert J. Complications of orthodontic-orthognathic surgery treatment in mentally handicap patients. J Craniomaxillofac Surg. 2006;34(3):156-161.

28. Bock JJ, Maurer P, Sterzik G, Schubert J. Possibilities and Limitations of Comprehensive Treatment of Mentally Retarded Patients with Cleft Lip, Alveolus and Palate. J Orofac Orthop 2004;65(3):259-268. 